\title{
Measurement of Pixel Number Variation Abnormality in Pursuit Eye Movement of Children with Autism
}

\author{
Ippei Torii", Takahito Niwa and Naohiro Ishii
}

Department of Information Science, Aichi Institute of Technology, Aichi, Japan

"Correspondence to:

Ippei Torii

Doctor (Business Administration and Computer

Science), Department of Information Science

Aichi Institute of Technology

Toyota, 470-0392, Japan

Tel: +81565488121

E-mail: mac@aitech.ac.jp

Received: February 13, 2017

Accepted: May 05, 2017

Published: May 09, 2017

Citation: Torri I, Niwa T, Ishii N. 2017. Measurement of Pixel Number Variation Abnormality in Pursuit Eye Movement of Children with Autism. J Neurol Exp Neurosci 3(1): 18-24.

Copyright: (C) 2017 Torri et al. This is an Open Access article distributed under the terms of the Creative Commons Attribution 4.0 International License (CC-BY) (http://creativecommons. org/licenses/by/4.0/) which permits commercial use, including reproduction, adaptation, and distribution of the article provided the original author and source are credited.

Published by United Scientific Group

\section{Abstract}

In Japan, the prevalence of autism, a condition that affects communication and social development, is 10 times higher than it was in the past. The number of children receiving special education in public elementary and junior high schools across Japan has surpassed 90,000, corresponding to an increase of 7.4-fold over the past 20 years (since 1993). The authors aimed to establish an objective diagnostic criterion for children with autism spectrum disorder. To this end, they conducted eye-tracking tests on children with autism and neurotypical children. Using a technique developed by one of the authors, which uses afterimages to determine eye blinking (Patent No. 5871290), they obtained the pixel number variation (a numerical value) in gaze direction based on the center of mass of pixels associated with the pupil. The results were then plotted onto a two-dimensional graph, and distributions based on probability density function and receiver operating characteristic curve analysis were ascertained. This analysis yielded a decision boundary clearly demarcating autism and neurotypical distributions and, thus, confirming the reliability of the method. The results revealed that autistic subjects failed to track the moving object. This finding suggests that this technique of measuring abnormality in pixel number is effective for distinguishing individuals with autism from those with typical development and, thus, can serve as an objective criterion for the diagnosis of autism. The assessment method enables the capture of eye movement based on afterimages without the need for any special equipment: only a camera-mounted personal computer is required. In other words, the technology provides a clear and simple method of detection and, as such, can serve as a supplementary assessment tool for psychiatrists in diagnosing autism in children. Moreover, it can also assess learning and treatment efficacy among individuals with autism in educational settings.

\section{Keywords}

Oculomotor, Autism, Kanner syndrome, ROC curve, Afterimage, Line-ofSignt detection

\section{Introduction}

Autistic spectrum disorder (ASD) is a developmental disorder; the number of patients has recently been increasing rapidly due to the influence of changes in diagnostic criteria. It is also difficult to distinguish the autistic spectrum from other developmental disorders such as learning disability. Therefore, a diagnostic tool that can assess the disorder objectively and quantitatively is required.

According to a 2014 report from the United States Centers for Disease Control and Prevention, as of 2010, 1 in 68 children had been identified with ASD. This estimate is approximately $30 \%$ higher than previous estimates 
reported in 2012 ( 1 in 88 children, based on data from 2008). The sharp rise in the prevalence of ASD is related to growing public awareness of autism. When parents seek a diagnosis at an early stage, it increases the chances of detecting autism. The causes of autism are unclear and, to date, there no effective treatment or preventive measures [1-4]. In Japan, approximately $10 \%$ of young boys and approximately $5 \%$ of girls have a developmental disorder. In 2003, the government proposed the Act for Support for Persons with Developmental Disabilities and, in 2006, commenced special needs education for children with disabilities [5, 6] (National Institute of Special Needs Education, JAPAN). In addition, the government proactively supports research activities related to developmental disabilities. Against this backdrop, there is a need for a simple, inexpensive, and quantitative diagnostic tool that anyone can use to diagnose a developmental disorder.

The fundamental mechanisms of developmental disability have yet to be elucidated; however, the number of diagnoses of disability appeared to increase after the Ministry of Health, Labor and Welfare began to support breast-feeding programs promoted by the United Nations Children's Fund (UNICEF and the World Health Organization (WHO) in 1993. When the Ministry of Health, Labor and Welfare recommended exclusive breastfeeding and kangaroo-mother care in 2007, the number of developmental disabilities increased rapidly; consequently, it is believed that those movements were significant factors [7-9] (Figure 1).

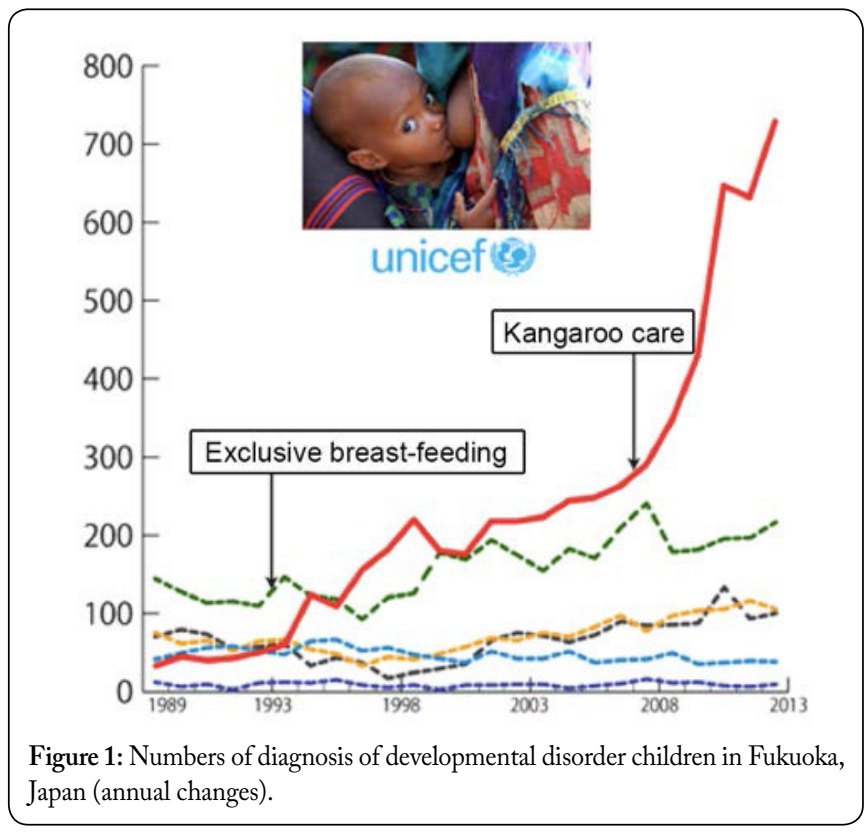

Excessive emphasis on exclusive breastfeeding could cause severe jaundice (hyperbilirubinemia), hypoglycemia, or dehydration (hypernatremia dehydration) because of nutritional deficiency. It has also been pointed out that hypothermia caused by sudden drops in body temperature can occur in kangaroo-mother care (early stage contact of a mother and child). It has been reported that there are few occurrences of children with disabilities at medical agencies adopting prevention measures for newborn hypothermia and hypoglycemia.
Secretion of breast milk is difficult immediately after childbearing, even for multiparous mothers. However, the joint statement from UNICEF and WHO for breast-feeding child care recommend the following:

- Help mothers initiate breastfeeding within $30 \mathrm{~min}$ of birth.

- Give newborn infants no food or drink other than breast milk, unless medically indicated.

- Practice "rooming in", i.e., allow mothers and infants to remain together $24 \mathrm{~h}$ per day.

- Train all health care staff in the skills necessary to implement this policy.

If the cause of the developmental disorder is hereditary, there should be no differences in incidence rates across geographical areas. However, the rate of developmental disorders in Kyoto, Yokohama, Nagoya, Kitakyushu, and Hiroshima is remarkably high (Figure 2).

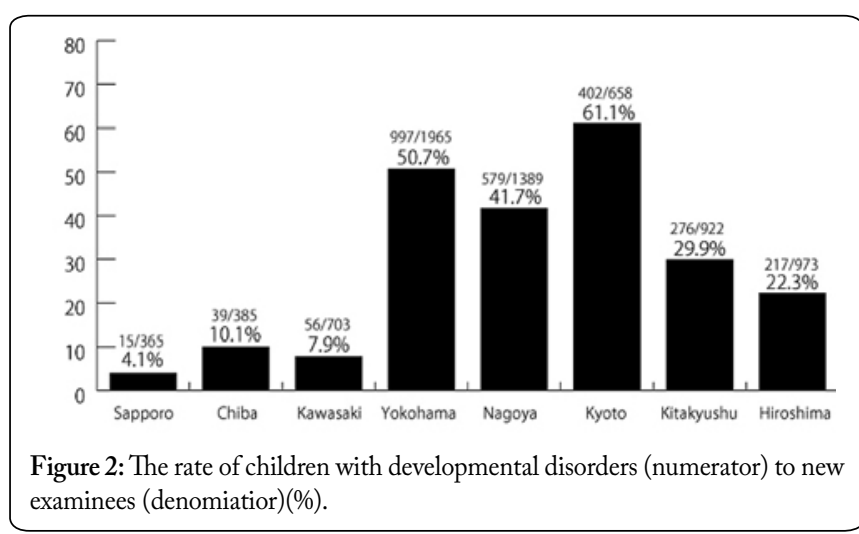

The system of special support services in resource rooms was elasticized by a change in School Education Law in April 2007; the Disability Discrimination Act was implemented in April 2016. While establishing these legal and governmental frameworks, a method for correct and early detection of a child with disabilities for appropriate support has been required. Assessments for developmental disabilities vary widely and depend on the subjective judgments of an evaluator. Diagnosis is entrusted to the judgment of experienced psychiatrists, who, furthermore, must also consider various confirmatory elements of the diagnosis. Therefore, a supplementary method of examination, with which anyone can obtain an objective quantitative assessment, is needed.

\section{Purpose of the Study}

One of the authors previously developed a technique for eye blink evaluation based on afterimages. In the present study, we used this method for measuring abnormalities in gaze direction based on variations in the center of mass of pixels associated with the pupil.

The procedure for measuring abnormality in gaze direction is as follows:

1. Identify the pixels associated with the pupil in the captured frames (detection device: Open CV, Haar-like); 
2. Prepare reference images of blink/gaze direction, known as afterimages, from the pupil pixels;

3. Place coordinates on the frame image;

4. Determine the center of mass of the pixels based on afterimages and position of the iris;

5. Subject views moving image for $10 \mathrm{~s}$; during these $10 \mathrm{~s}$, record coordinate changes in center of mass

6. Process camera images with a precision of 30-40 frames/s and convert the amount of change in center of mass of the pixels into numerical values.

We plotted the values yielded from the above procedure onto a graph, and then compared the typical development and autism groups using a probability density function. Based on the compared data, we calculated the decision boundary and used receiver operating characteristic (ROC) curve analysis, which confirmed the reliability of our assessment method. Thus, we developed an objective criterion that can distinguish individuals with typical (i.e., normal) development from those with autism accurately and early

\section{Structure of the System}

The difference in the area of the extent of change occurs by measuring the difference between the afterimage in consecutive frames and the present frame. We set the amount of change to the quantity

of eyeball movements. This method made it possible to detect and quantify changes in eyeball movements in numerical values. The difference in these movements between non-autistic and autistic individuals was compared and analyzed. Figure 3 is an illustration indicating the states to assess opening and closing of the eyes.

Images are always assessed consecutively. We assigned each pixel a persistence value (p), and subtracted 1 from every frame $p$. Each $p$ is an integer of zero or above. We added 5 to the $\mathrm{p}$ of pixels that we deemed to be black in color.

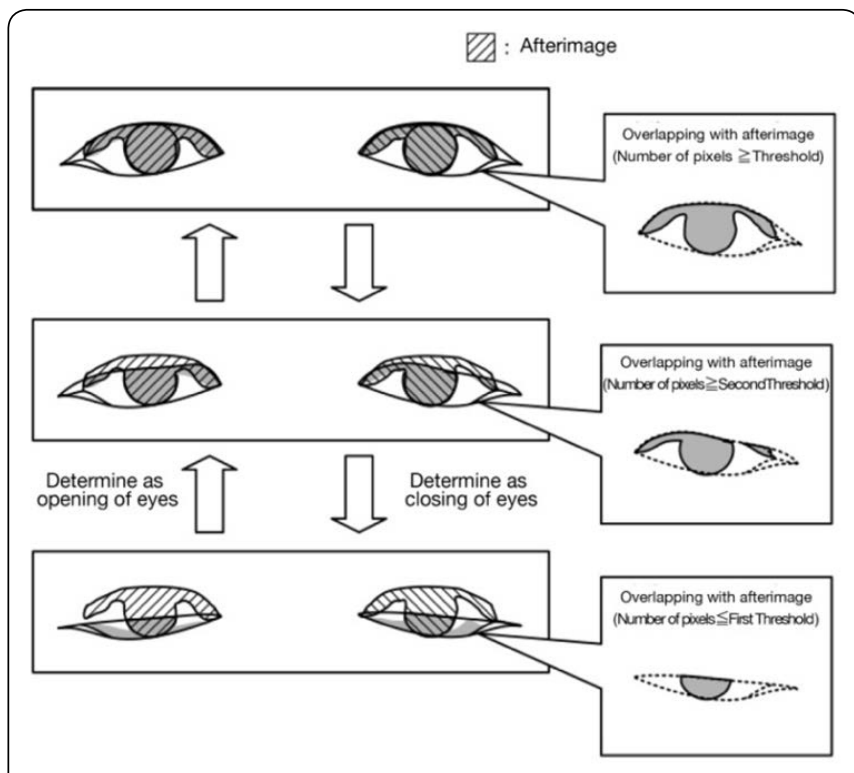

Figure 3: Diagram of opening and closing of eye
We define the black part as an afterimage based on value of image persistence. The value becomes - $1+5$ when the value of image persistence is judged black, and the program is forced to consider it an afterimage when the value is $>50$. More than 50 means the part that is judged to be black during approximately $0.45 \mathrm{~s}$ is determined as an afterimage because 24 to 30 frames are processed per second (Figure 4).

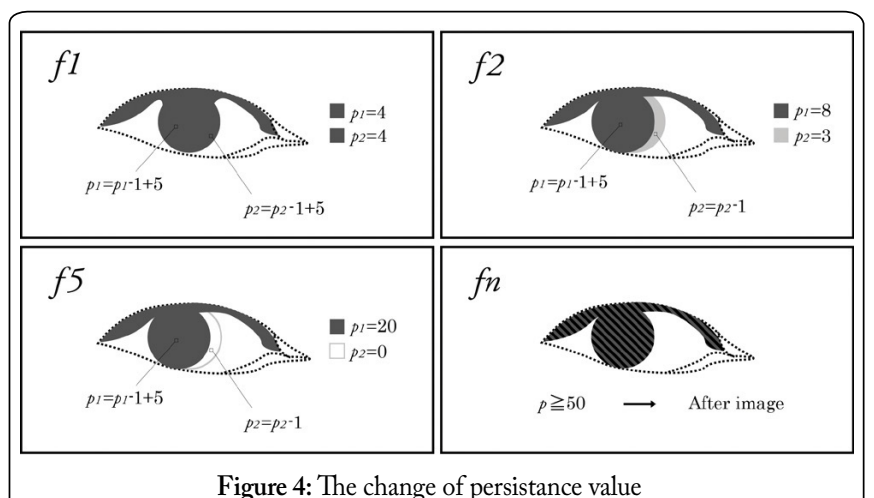

To detect gaze direction, there are methods using circles or ovals focused on the pupils. However, even in healthy individuals, pupils cannot be visualized unless the eyes are wide open (Figure 5).

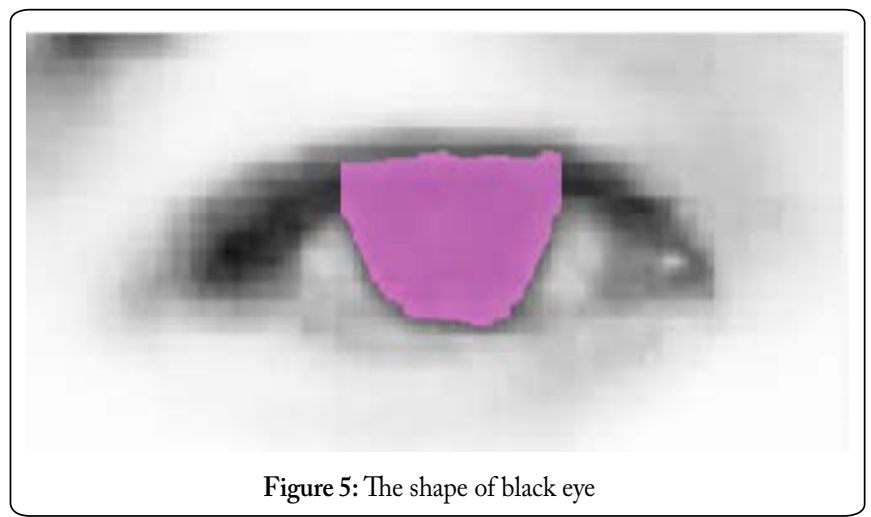

These techniques are not suitable for physically handicapped individuals who often cannot open their eyes sufficiently. Therefore, by considering the center of gravity of the eye and comparing it with the afterimage, we developed a new method to detect gaze direction. Using this gaze direction detection method, the average change in the pixel center of gravity of the eye movement per minute in the subject is determined and analyzed, thus distinguishing normally developing children and those with Kanner syndrome.

To define the center of gravity, the acquired eye region is binarized. Generally, the first moment of circumference of the center of gravity is zero. In other words, it refers to the point where weights are balanced; therefore, this was used to calculate the center of gravity (Figure 6).

It is difficult to accurately grasp the center of the pupil using this pixel center of gravity. However, compared with circular or oval detection methods, the image processing speed is significantly faster. Additionally, even when the shape of 
the pupils collapses due to blurring of the gaze, the center of gravity can always be captured. After defining the center of gravity of the pixel, movements of the pupils are detected for each frame (Figure 7).

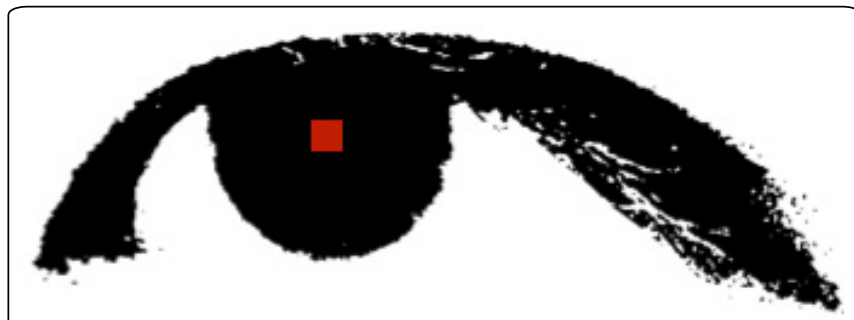

Figure 6: The center of gravity

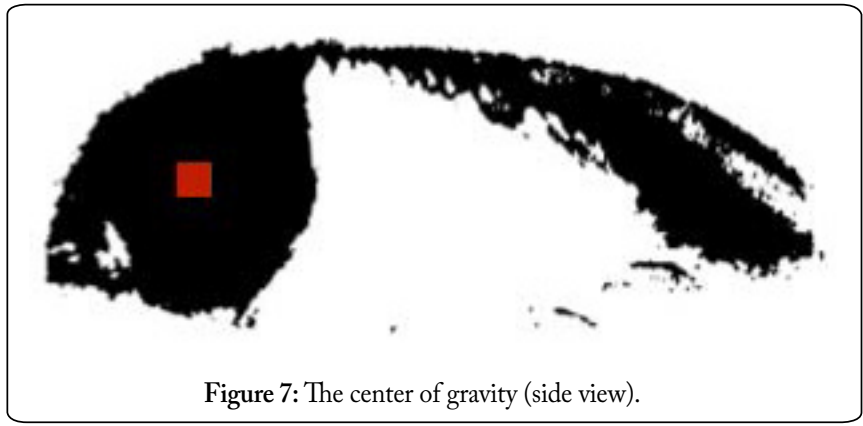

In the judgment method, pixel values on the $\mathrm{x}$ axis and $y$ axis are generated from the upper left to the lower right of the image when cutting the eye part image in the first image processing. The centroid of the afterimage with the $x$ coordinate of the current frame is compared and the amount of change in coordinates between frames is recorded. This corresponds to the vibration of eye movement (Figure 8).

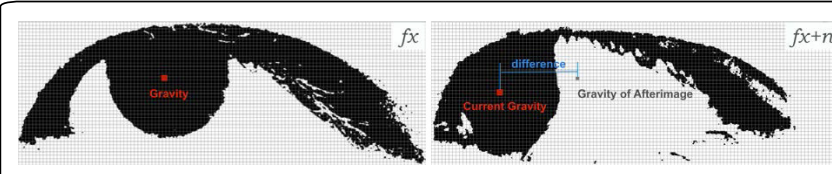

Figure 8: Comparison of afterimage and current frame.

The average change in the quantity of this numerical value over a span of $1 \mathrm{~min}$ is defined as fine movement of the eyeball, and it is used to distinguish individuals in a normally developing group from those in a Kanner syndrome group (Figure 9).

\section{Clinical Application to Children with Autism using Pixel Number Variation Abnormality}

The research team from Osaka University has conducted several studies investigating eye movement abnormalities in children with attention deficit hyperactivity disorder (ADHD) using the Tobii eye tracker to measure saccadic ocular movement [10-13]. The diagnosis of such an abnormality was based on a comparison of the time the subject spent fixing their gaze on an object [14-16], but there is no link to results.

Circular movements that combine saccade and pursuit are shown in the experiment so that a child with autism could gaze. A circle that begins to move from the left moves suddenly out of the way and appears at the right. To use this as an evaluation index of autism, a method to compare and analyze data from the eyes captured by a camera in the $\mathrm{PC}$ to establish a density function was built. Subjects in the analysis included 37 elementary school autistic children, 15 elementary school normally developing children, 10 junior high school autistic children, 10 junior high school normally developing children, 7 high school autistic children and 5 high school normally developing children. The calculated average of the amount of change in eyeball movement with eye shift between frames was compared and analyzed. The reliability of this analysis method was measured using ROC curve analysis and box-and-whisker plots.

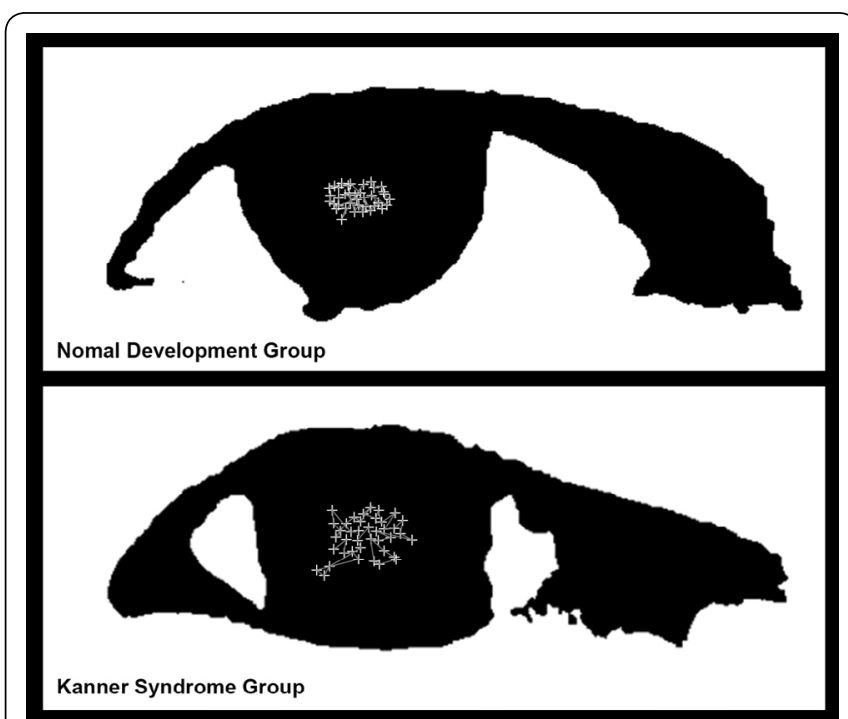

Figure 9: Difference in blur in line-of-sight detection between normal development group and kanner syndrome group. detect blur and compare the numbers

We measured the number of pixels that diverged from the trajectory of the afterimages, and calculated the mean values for changes in the amount of movement of both eyes. Figure 10 is a scatterplot illustrating the degree of dispersion in eye movement in each group. In the case of the normally

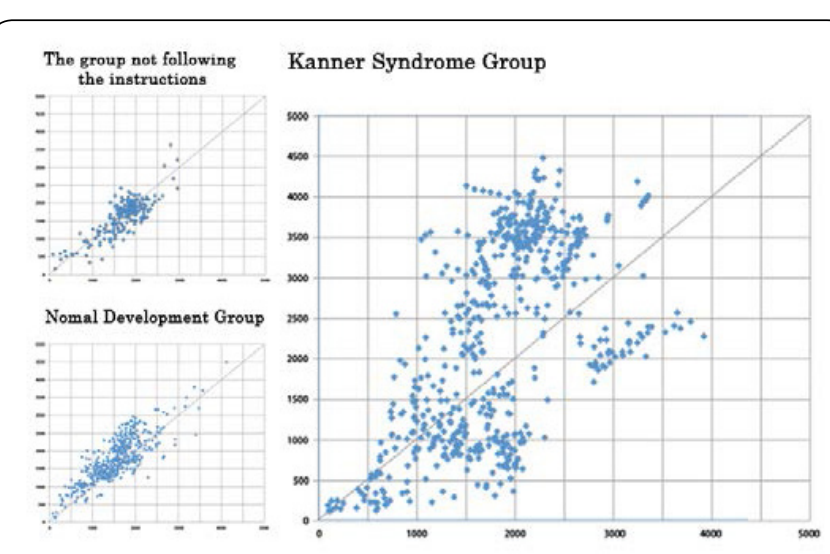

Figure 10: Difference of eyes dispersion between normal developing group and autism group. 
developing group, the points are clustered around a central line. In the autism group, however, these points are dispersed.

This finding indicates that the normally developing subjects could track the target, the autism subjects could not. The data pertaining to the autism group exhibited its independence when compared with data pertaining to children and adults with typical development. Comparison of data obtained from 30 normally developed individuals in this study based on a probability density function yielded a clear decision boundary.

Figure 11 shows the relationship between the typicaldevelopment group, the autism group, and the unable-tofollow-instructions group in terms of probability density function. The red line indicates the unable-to-followinstructions group, the blue line indicates the typicaldevelopment group, and the green line indicates the autism group. This figure provides further confirmation of the independence of each group's data.

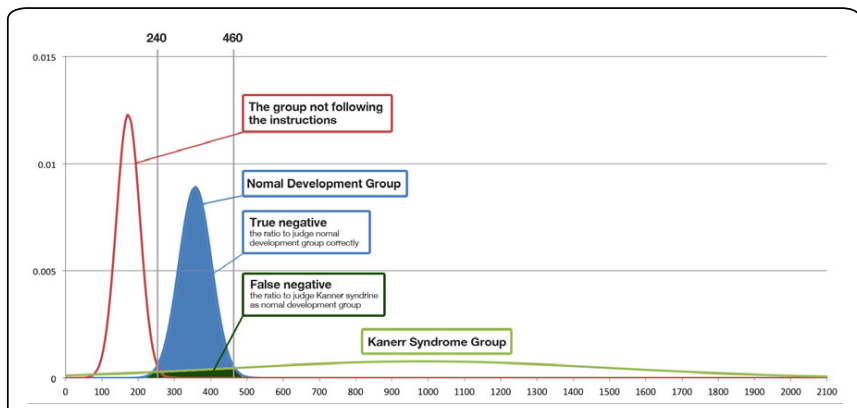

Figure 11: Relations of normal developing group and the autism group with the density of probability.

From these results, the identification border between the unable-to-follow-instruction group and the normally developing group was 240 . The border between the autism group and the normally developing group was 460 .

Figure 12 is a chart that applied the average changes of the three groups to a box-and-whisker plot. While the lower quartile is within 300 and the upper quartile is within 400 in the normally developing group, the lower quartile is 550 and upper quartile is 1100 in the autism group, with a wide

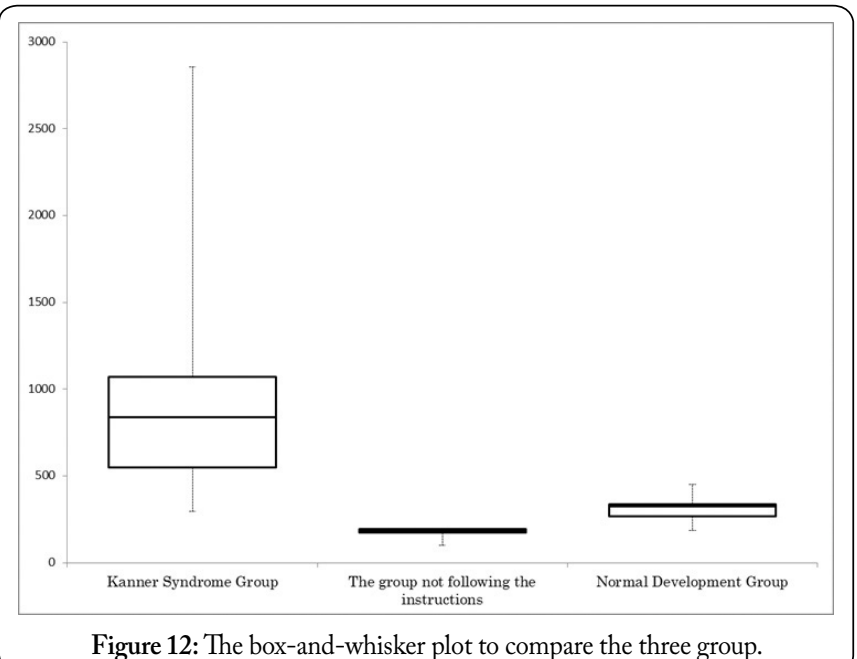

Figure 12: The box-and-whisker plot to compare the three group. dispersion of data. The lower quartile of the unable-to-followinstruction group was 170 and the upper quartile was 192: the range and dispersion were both very small. It was found that each data point in this graph was peculiar.

The detection technique developed in this research should be effective in distinguishing children with autism from normally developing children (Figure 12).

Based on these results, a new system to measure ocular movements of subjects in PEM (Pursuit eye movement) precisely is being developed. A movie in which points of gaze are indicated at regular intervals (movie without gap) are shown to a subject and he/she is directed to watch the points as closely as possible. Next, the points of gaze are indicated at regular intervals, where they disappear and then appear again (movie with gap). A gap in a point of gaze flashing on and off at random removes against prediction. When a point was shown the second time, the eye movements of saccade of subjects in the normal development group became more rapid than usual, but those in the autism group did not. Comparing reaction times (reaction latency) according to age, the autism group exhibited a delay in reactions in both movies with and without gap. This suggests an abnormality in the root or the function to maintain involuntary gazing activity and to start a pursuit movement in the ocular movement controlling mechanism in the brains of children with ASD.

As the result of this experiment, delays in following objects with the eyes were observed in autistic children compared with normally developing children with or without gap; however, the delay tends to happen conspicuously with gap. To date, there have been no studies that have reported such an abnormality in the gap effect, which is the ability to correspond to a predicted movement in autism spectrum. Therefore, this result can be expected to apply clinically as well as be useful to solute decease states of a nervous foundation in the brain (Figure 13).

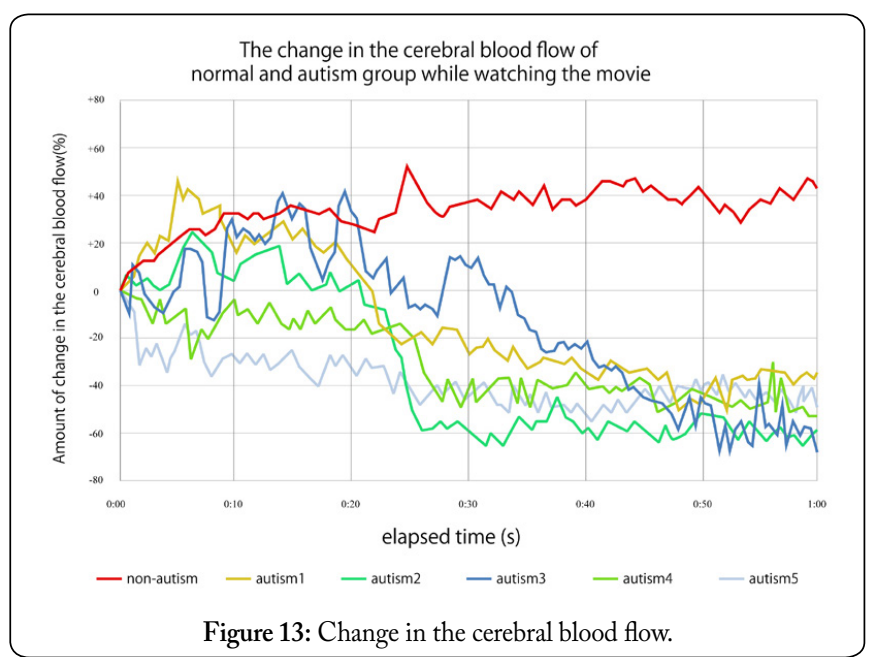

Cerebral blood flow in normally developing children exhibits constant increases. In contrast, the cerebral blood flow of children with autism falls suddenly because he/she cannot follow the quick movements of points of gaze and the line of sight deviates. 
Figure 14 is a truth value table showing the probabilities for positives and negatives. The probability for accurately determining typical development was 99\%, and the probability of a false positive (i.e., the probability that the subject is developing normally but is actually autistic) is $1 \%$. The probability for accurately determining autism is $96 \%$, and the probability of a false negative (i.e., the probability that the subject is autistic but is actually neurotypical) is less than $4.0 \%$.

\begin{tabular}{|c|c|c|}
\hline & FALSE & TRUE \\
\hline Negative & False Negative Rate & True Negative Rate \\
$(\leqq 240<480)$ & 0.04 & 0.99 \\
\hline Positive & False Positive Rate & True Positive Rate \\
$(480 \leqq)$ & 0.01 & 0.96 \\
\hline
\end{tabular}

Figure 14: Boolean table indication whether positive or negative.

The reliability of the results is measured using ROC curve analysis $[17,18]$. The reliability that could distinguish a normal developing individual from an autistic one is $98.77 \%$ (Figure 15).

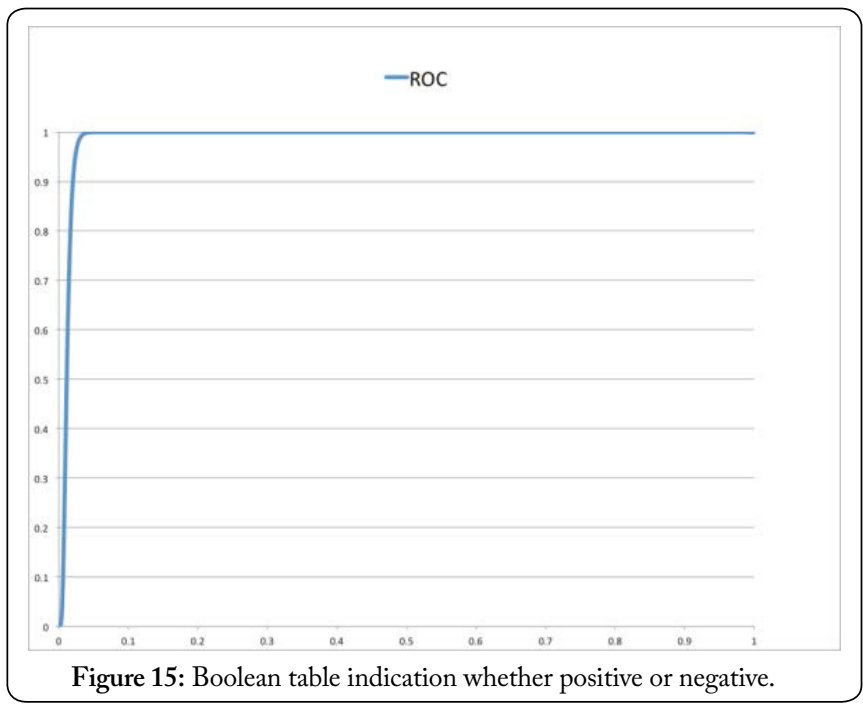

On ROC analysis, the shape shifts to the top left where there is a small peak of two density functions, and shows that the performance of the decision surface line is high. The area under the ROC curve (AUC) was 0.9877; the closer to 1 the AUC is, the more reliable the test is. The AUC we obtained, therefore, suggests that the device produced false readings in $<2 \%$ of cases. Thus, our method for detecting autism exhibits excellent accuracy.

\section{Conclusion}

In this study, we aimed to develop a technique for measuring pixel number variation in gaze direction based on the center of mass of pixels associated with the pupil. The ultimate aim was to establish an objective criterion that physicians can use to corroborate subjective diagnoses of ASD. Using a camera-mounted PC, we developed a high-precision image processing technique to establish an objective criterion that can distinguish individuals with autism from those with normal development.

This measurement technique does not burden the subject; it can obtain data in $15 \mathrm{~s}$. The technique is unique in that it indicates the potential of ASD without the need for observing the individual's behavior. As such, it is effective for identifying children with disorders and will in turn enable the preparation of measures and educational approaches that cater to their needs. We hope that the precision of this tool will be further refined in the future, and that it will be used broadly as a simple and accessible tool for quantitatively assessing ASD.

\section{Author Contributions}

IP conducted research on the subjects and development of the device. He was also the primary author of this article. TN processed the acquired data; he also assisted in writing this article. NI was responsible for verifying the validity of the research method and the data. All authors have read and approved the final manuscript.

\section{References}

1. Kitazawa S.2005.Where tactile stimuli are ordered in time."Probabilistic mechanisms of learn-ing and development in sensorimotor systems" ESF-EMBO symposia on "Three-Dimensional Sensory and Motor Space", San Feliu de Guixols, Spain.

2. Kitazawa S. 2006. Reversal of subjective temporal order due to sensory and motor integrations. In: P. Haggard, M. Kawato, Y. Rossetti (eds) Sensorimotor foundations of higher cognition (Attention and Performance XXII) Oxford University Press, Chateau de Pizay, France, pp 73-97.

3. Kitazawa S. 2007. Reversal of subjective temporal order due to eye and hand movements. ESF-EMBO symposia on Three-Dimensional Sensory and Motor Space, SantFeliu de Guixols, Spain.

4. Kitazawa S, Nishida S. 2008. Adaptive anomalies in conscious time perception. Tutorial workshop in the $12^{\text {th }}$ Annual Meeting of the Association for the Scientific Study of Consciousness. Taipei, Taiwan.

5. Ministry of Health, Labour and Welfare: Guidelines about person of development child with a disability support and the assessment, 2013.

6. Guidelines about person of development child with a disability support and the assessment, 2012.

7. Takahashi N. 2006. Symptomatic hypoglycemia in a breastfed neonate. The Journal of the Japan Pediatric Society 110(6): 789-793.

8. Watabe S. 2011. Kangaroo mother care and skin-to-skin. Journal of Japan Society of Perinatal and Neonatal Medicine 47(4): 731-735.

9. Kubota S. 2009. The delivery room in japan is too cold. The Journal of the Japanese Society for Breastfeeding Research 3: 70-74.

10. Nakano T, Tanaka K, Endo Y, Yamane Y, Yamamoto T, et al. 2010. Atypical gaze patterns in children and adults with autism spectrum disorders dissociated from developmental changes in gaze behaviour. Proc Biol Sci 277(1696): 2935-2943. https://doi.org/10.1098/ rspb.2010.0587

11. S. Kitazawa. Eye tracking research.

12. Nakano T, Ota H, Kato N, Kitazawa S. 2010. Deficit in visual temporal integration in autism spectrum disorders. Proc R Soc B 277(1684): 10271030. https://doi.org/10.1098/rspb.2009.1713

13. Kitazawa S. 2010. Towards the understanding and treatment of autism. In: Ota J, Aonuma H (eds) Dysfunction (series Mobiligence) and volume 4 expression social adjustment mechanism, Ohmsha Ltd., Tokyo, Japan, pp 213-254. 
14. KimuraY, Kobayashi H. Clinical study on movement education application of autistic children- approach to enhance the Motor Imitation. In: The $25^{\text {th }}$ Japan special education society papers, pp 446447.

15. Iwanaga R, Kawasaki C. For sensorimotor disorder of high- functioning autism children. Children of the spirit and nerve 36(4): 27-332.

16. Fukuda S, Okamoto M, Kato K, Murata E, Yamamoto T, et al. 2011. A perception of the others' gaze for children with pervasive developmental disorders. Hum Dev Res 25: 135-148.
17. Kawashima H, Hayashi N, Ohno N, Matsuura Y, Sanada S. 2015. Comparative study of patient identifications for conventional and portable chest radiographs utilizing ROC analysis. J Radiat Res 71(8): 663-669.https://doi.org/10.6009/jjrt.2015_JSRT_71.8.663

18. Kitashoji E, Koizumi N, Lacuesta TLV, Usuda D, Ribo MR. 2015. Diagnostic accuracy of recombinant immunoglobulin-like protein A-based IgM ELISA for the early diagnosis of Leptospirosis in the Philippines. PLoS Negl Trop Dis 9(6): e0003879. https://doi. org/10.1371/journal.pntd.0003879 